\title{
Review of: "Variation in fine root traits with thinning intensity in a Chinese fir plantation insights from branching order and functional groups"
}

Silong Wang

Potential competing interests: The author(s) declared that no potential competing interests exist.

This is an interesting study, and this study found the acclimation mechanism of fine roots to forest thinning, a very common management practices. In this study, the fine roots were differentiated into two functional groups, absorptive and transport roots. The absorptive root traits of Chinese fir were more sensitive to thinning than transport roots. Importantly, the study found that the absorptive roots of Chinese fir changed from higher $\mathrm{N}$ and $\mathrm{C}$ concentration and low abundance to lower $\mathrm{C}$ and $\mathrm{N}$ concentration and higher abundance with increasing thinning intensity. These suggest that the absorptive roots of Chinese fir take two-dimensional strategies to acclimate to the altered surrounding after thinning. Particularly, this study unraveled the mechanism of interaction of understory development and soil nutrients after thinning. Meanwhile, this paper has some shortage in the exact explanation in the discusion and description of the mechanism of acclimation strategies (figure 5)with English language. In the second paragraph of discusion section, the sentence "Converstly, light decreased with decreaseing thinning intensity....." the word "lihgt" doestn't make clear sense here, is it received by the individual canopy? Secondly, in the upper part the figure 5, from lower thinning to higher thinning intensity, there is the description of soil quality, "rich-soil quality" and "poor-soil quality" are not appropriate here, because it is not possible for the thinning treatment to alter the soil quality from rich-soil to poor-soil. 